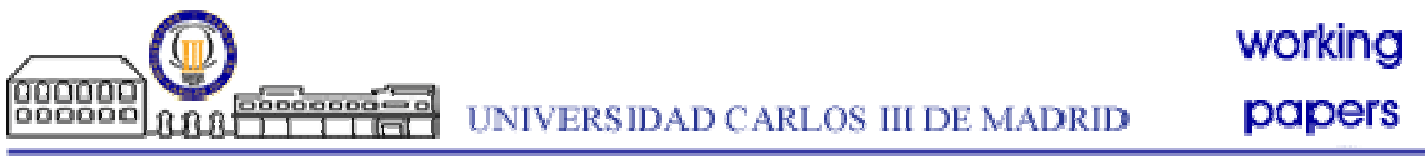

Working Paper 07-03

Departamento de Estadística

Statistic and Econometric Series 01 Universidad Carlos III de Madrid

January 2007

Calle Madrid, 126

28903 Getafe (Spain)

Fax (34-91) 6249849

\title{
ESTIMATING THE SYSTEM ORDER BY SUBSPACE METHODS*
}

\author{
Alfredo García-Hiernaux, ${ }^{1}$ José Casals ${ }^{2}$ and Miguel Jerez ${ }^{3}$
}

\begin{abstract}
This paper discusses how to determine the order of a state-space model. To do so, we start by revising existing approaches and find in them three basic shortcomings: i) some of them have a poor performance in short samples, ii) most of them are not robust and iii) none of them can accommodate seasonality. We tackle the first two issues by proposing new and refined criteria. The third issue is dealt with by decomposing the system into regular and seasonal sub-systems. The performance of all the procedures considered is analyzed through Monte Carlo simulations.
\end{abstract}

Keywords: System order, State-space models, subspace methods, information criteria, seasonality

\footnotetext{
* This research was supported by the Spanish Ministry of Education and Science, under grant SEJ200507388.

1 Department of Statistics, Universidad Carlos III de Madrid, Campus de Getafe, 28903 Madrid (SPAIN). email: aghierna@est-econ.uc3m.es

2 Department of Quantitative Economy, Universidad Complutense de Madrid

3 Department of Quantitative Economy, Universidad Complutense de Madrid
} 


\section{Introduction}

The order of a linear system is the number of dynamic components that must be combined to represent the data dynamics. Determining this value, also known as McMillan index, is critical in applied data modelling and, accordingly, there is an extensive literature about this subject which can be broken into two broad categories: i) preliminary data analysis, or a priori methods, and ii) model comparison procedures, also known as a posteriori methods.

The first group includes very different proposals. Some of them check whether the model dimension is too high using the condition number of the information matrix, see Stoica and Söderström (1982). Other authors, Cooper and Wood (1982), Tsay (1989), Tiao and Tsay (1989), estimate the system order using canonical correlations. In a recent paper, Toscano and Reisen (2000) revise and compare these methods. Aoki (1990) applies the same technique to state space models. Close to this approach, Bauer (2001) presents a subspace-based criterion to determine the system order, while Sorelius (1999) does it by testing the rank of a particular sample covariance matrix, see also Woodside (1971). Finally, other authors apply spectral techniques to fit an empirical transfer function, which points out what system orders are required to describe the data dynamics, see e.g., Wei (1990).

A posteriori methods typically rely on information criteria. Broadly speaking, they compare several alternative models by balancing their data fit, measured through the log-likelihood, with a penalty that depends on the sample size and a measure of model complexity. The pioneer criterion in this line is due to 
Akaike (1976), which seminal work motivated many other proposals such as those of Schwartz (1978) and Hannan and Quinn (1979).

When applied to economic time series, all these procedures share a basic shortcoming: they were not originally designed to cope with seasonality. On the other hand, Monte Carlo experiments reveal two additional weaknesses: (a) many procedures are not robust, meaning that their ability to choose the right system order depends critically of the dynamics of the data; and (b) some methods display a poor performance in short samples. According to this diagnostic, the contribution of this paper is threefold. First, we propose two refinements of the criteria suggested by Bauer (2001), designed to improve its finite-sample performance. Second, we present a simple automatic procedure that has a clear advantage in terms of robustness when compared with other methods. Last, we extend the use of any criterion to determine the order of the seasonal and regular sub-systems in multiplicative seasonal processes. The performance of all these proposals is analyzed through Monte Carlo simulations.

The paper is structured as follows. Section 2 defines the notation and introduces subspace estimation. The main order-determination procedures are reviewed in Section 3. Section 4 presents the main results of the paper. In Section 5 we discuss the performance of several methods by means of a simulation study. Finally, Section 6 provides some conclusions and indicates how to obtain a free MATLAB toolbox that implements all the algorithms proposed. 


\section{Model set and subspace estimation algorithms}

Consider a linear fixed-coefficients system that can be described by the state space (SS) model,

$$
\begin{aligned}
\boldsymbol{x}_{t+1} & =\boldsymbol{\Phi} \boldsymbol{x}_{\boldsymbol{t}}+\boldsymbol{E} \psi_{t} \\
z_{t} & =\boldsymbol{H} \boldsymbol{x}_{t}+\psi_{t}
\end{aligned}
$$

where $\boldsymbol{x}_{\boldsymbol{t}} \in \mathbb{R}^{n}$ is a state vector and $n$ denotes the true order of the system. $\boldsymbol{z}_{\boldsymbol{t}} \in \mathbb{R}^{m}$ is an observable output vector, $\boldsymbol{\psi}_{\boldsymbol{t}} \in \mathbb{R}^{m}$ is a white noise vector such that $\boldsymbol{\psi}_{\boldsymbol{t}} i i d N(\mathbf{0}, \boldsymbol{Q})$ and $\boldsymbol{\Phi} \in \mathbb{R}^{n \times n}, \boldsymbol{E} \in \mathbb{R}^{n \times m}$ and $\boldsymbol{H} \in \mathbb{R}^{m \times n}$ are the parametric matrices of the system. The system is assumed to be stable and strictly minimumphase, so that all the eigenvalues of $\boldsymbol{\Phi}$ and $(\boldsymbol{\Phi}-\boldsymbol{E} \boldsymbol{H})$ lie inside the unit circle.

Many SS models have different errors in the transition and observation equations, as well as a coefficient matrix affecting the observation error. The innovations model (1a)-(1b) does not conform to this common specification, but is general in the sense that any fixed-coefficients SS model can be written in this specific form (see, Casals et al., 1999, Theorem 1). Also, no generality is lost by assuming that the model has no observable inputs, since any model with inputs can be decomposed into an input-related and an error-related model (see e.g., Chui and Chen, 1999).

Subspace methods derive from the representation (1a-1b) in matrix form. By 
recursive substitution in (1a) we obtain,

$$
\boldsymbol{x}_{\boldsymbol{t}}=\boldsymbol{\Phi}^{t} \boldsymbol{x}_{\mathbf{0}}+\sum_{j=0}^{t-1} \boldsymbol{\Phi}^{j} \boldsymbol{E} \psi_{t-j-1}
$$

and substituting (2) into the observation equation (1b), we get:

$$
\boldsymbol{z}_{\boldsymbol{t}}=\boldsymbol{H} \boldsymbol{\Phi}^{\boldsymbol{t}} \boldsymbol{x}_{\mathbf{0}}+\boldsymbol{H} \sum_{j=0}^{t-1} \boldsymbol{\Phi}^{j} \boldsymbol{E} \psi_{t-j-1}+\boldsymbol{\psi}_{\boldsymbol{t}}
$$

so the endogenous variable, $\boldsymbol{z}_{\boldsymbol{t}}$, depends on the initial state vector, $\boldsymbol{x}_{\mathbf{0}}$, and the present and past innovations, $\boldsymbol{\psi}_{\boldsymbol{t}}$. Equation (3) can be written in matrix form as,

$$
Z_{i}=O_{i} X_{0}+V_{i} \Psi_{i}
$$

where the subscript $i$ is an integer that denotes the dimension of the row space of $\boldsymbol{Z}_{\boldsymbol{i}}$. This parameter may be set by the user or automatically chosen, depending on the specific subspace algorithm applied. In this work, we determine it using the rule $i=\max \left(4, h_{t}\right)$, being $h_{t}$ the nearest integer to $\log T$. This is an heuristic rule supported by empirical experience, but our methods can be used with any other criterion to determine $i$.

Equation (4) requires the following matrices related to the data:

1) Block-Hankel Matrices (BHM): The dimension of the row space of these matrices are denoted by $p$ and $f$, which stand, respectively, for the past and the future. The choice of these integers is discussed by Viberg (1995) and Peternell 
et al. (1996). Here, for convenience and simplicity, we assume that $p=f=i$. Under these conditions, the output BHM would be given by:

$$
Z_{p}=\left(\begin{array}{cccc}
z_{0} & z_{1} & \ldots & z_{T-2 i} \\
z_{1} & z_{2} & \ldots & z_{T-2 i+1} \\
\vdots & \vdots & & \vdots \\
z_{i-1} & z_{i} & \ldots & z_{T-i-1}
\end{array}\right) ; \quad Z_{f}=\left(\begin{array}{cccc}
z_{i} & z_{i+1} & \ldots & z_{T-i} \\
z_{i+1} & z_{i+2} & \ldots & z_{T-i+1} \\
\vdots & \vdots & & \vdots \\
z_{2 i-1} & z_{2 i} & \ldots & z_{T-1}
\end{array}\right)
$$

where $\boldsymbol{z}_{\mathbf{0}}$ is a vector of $m$ components. In (4), $\boldsymbol{\Psi}_{\boldsymbol{i}}$ is as $\boldsymbol{Z}_{\boldsymbol{i}}$ but with $\boldsymbol{\psi}_{\boldsymbol{t}}$ instead of $\boldsymbol{z}_{\boldsymbol{t}}$.

2) The state sequence, defined as $\boldsymbol{X}_{\boldsymbol{t}}=\left(\begin{array}{llllll}\boldsymbol{x}_{\boldsymbol{t}} & \boldsymbol{x}_{\boldsymbol{t}+\mathbf{1}} & \boldsymbol{x}_{\boldsymbol{t + 2}} & \ldots & \boldsymbol{x}_{\boldsymbol{t + T - 2 i}}\end{array}\right)$. The past and future state sequences beginning, respectively, at $t=0$ and $t=i$, can also be written as $\boldsymbol{X}_{\boldsymbol{p}}=\boldsymbol{X}_{\mathbf{0}}$ and $\boldsymbol{X}_{\boldsymbol{f}}=\boldsymbol{X}_{\boldsymbol{i}}$.

On the other hand, equation (4) includes also the following matrices related to the parameters in model $(1 \mathrm{a}-1 \mathrm{~b})$ :

3) The Extended Observability Matrix, which is:

$$
\boldsymbol{O}_{\boldsymbol{i}}=\left(\begin{array}{lllll}
\boldsymbol{H}^{\prime} & (\boldsymbol{H} \boldsymbol{\Phi})^{\prime} & \left(\boldsymbol{H} \boldsymbol{\Phi}^{2}\right)^{\prime} & \ldots & \left(\boldsymbol{H} \boldsymbol{\Phi}^{i-1}\right)^{\prime}
\end{array}\right)^{\prime} \in \mathbb{R}^{i m \times n}
$$


4) The lower block triangular Toeplitz matrix, defined as:

$$
V_{i}=\left(\begin{array}{ccccc}
\boldsymbol{I}_{m} & 0 & 0 & \ldots & 0 \\
H E & \boldsymbol{I}_{m} & 0 & \ldots & 0 \\
H \Phi E & H E & I_{m} & \ldots & 0 \\
\vdots & \vdots & \vdots & \vdots & \vdots \\
H \Phi^{i-2} E & H \Phi^{i-3} E & H \Phi^{i-4} E & \ldots & \boldsymbol{I}_{m}
\end{array}\right) \in \mathbb{R}^{i m \times i m}
$$

Due to the linearity of the system, the future state sequence can be written as $\boldsymbol{X}_{\boldsymbol{f}}=\boldsymbol{M} \boldsymbol{Z}_{\boldsymbol{p}}$, where the rank of $\boldsymbol{M}$ is $n$, the true system order. Therefore, shifting time subscripts in (4) and, substituting $\boldsymbol{X}_{\boldsymbol{f}}$, by $\boldsymbol{M} \boldsymbol{Z}_{\boldsymbol{p}}$, we obtain,

$$
Z_{f}=O_{i} M Z_{p}+V_{i} \Psi_{f}
$$

where $\boldsymbol{Z}_{\boldsymbol{f}}, \boldsymbol{Z}_{\boldsymbol{p}}$ and $\boldsymbol{\Psi}_{\boldsymbol{f}}$ are as in (5), and $\boldsymbol{O}_{\boldsymbol{i}}$ and $\boldsymbol{V}_{\boldsymbol{i}}$ are respectively as in (6) and (7).

Subspace methods estimate $\boldsymbol{O}_{\boldsymbol{i}}, \boldsymbol{M}$ and $\boldsymbol{V}_{\boldsymbol{i}}$ in (8) by solving a reduced-rank weighted least square problem. This is typically done by means of the Singular Value Decomposition (SVD, Eckart and Young, 1936) of the product $\boldsymbol{W}_{\mathbf{1}} \boldsymbol{Z}_{\boldsymbol{f}} \boldsymbol{W}_{\mathbf{2}}$, being $\boldsymbol{W}_{\mathbf{1}}$ and $\boldsymbol{W}_{\mathbf{2}}$ two weighting matrices (see, e.g., Katayama, 2005). Then, an estimation of the parameter matrices in (1a-1b) can be straightforwardly obtained from $\boldsymbol{O}_{\boldsymbol{i}}, \boldsymbol{M}$ and $\boldsymbol{V}_{\boldsymbol{i}}$. Computing the SVD requires specifying the matrices $\boldsymbol{W}_{\mathbf{1}}, \boldsymbol{W}_{\mathbf{2}}$. The literature provides several methods to solve this issue. For instance, Larimore-type methods (Larimore, 1983, 1990) use the weighting matrices $W_{1}=\left(Z_{f} Z_{f}^{\prime}\right)^{-\frac{1}{2}}$ and $W_{2}=Z_{p}^{\prime}\left(Z_{p} Z_{p}^{\prime}\right)^{-1} Z_{p}$. In this work, we will also consider the weighting matrix $\boldsymbol{W}_{\mathbf{1}}=\left(\tilde{\boldsymbol{Z}}_{\boldsymbol{f}} \tilde{\boldsymbol{Z}}_{f}^{\prime}\right)^{-\frac{1}{2}}$, where $\tilde{Z}_{f}$ are the residuals of regressing 
$Z_{f}$ onto $Z_{p}$ in a previous step.

\section{An overview of some specification procedures}

Estimating the dimension of the state vector, $n$, is not trivial, in particular when the sample is short. In this section we describe some criteria and test statistics that can be applied to this purpose, distinguishing between a priori and a posteriori methods. The difference between both approaches is in the need of estimating a model: the latter requires to do so while the former does not.

\subsection{A priori methods}

Tsay (1989) approaches order determination by testing the null hypothesis that the smallest canonical correlations $\hat{\sigma}_{n+1}, \hat{\sigma}_{n+2}, \ldots, \hat{\sigma}_{i m}$ are zero. To do so, he suggests the statistic $D(n)$, that can be straightforwardly adapted to subspace methods as:

$$
D(n)=-(T-2 i+1) \sum_{k=n+1}^{i m} \log \left(1-\frac{\hat{\sigma}_{k}^{2}}{\hat{d}_{k}}\right)
$$

with

$$
\hat{d}_{k}=1+2 \sum_{l=1}^{i} \hat{\rho}_{p k}(l) \hat{\rho}_{f k}(l)
$$

where $\hat{\rho}_{f k}(l)$ and $\hat{\rho}_{p k}(l)$ are the sample $l$-order autocorrelations of the canonical variables (see Tsay, 1989). Subscripts $p$ and $f$ refer to the past and future blocks

respectively. The term $\hat{d}_{k}$ is related to the asymptotic variance of the estimated canonical correlation coefficients in a context of serial correlation. Tsay (1989) proves that, under $H_{0}: \hat{\sigma}_{n+1}=\hat{\sigma}_{n+2}=\ldots=\hat{\sigma}_{i m}=0$, the statistic $D(n)$ follows a 
$\chi^{2}$ distribution with $2(i m-n)$ degrees of freedom.

In a subspace framework, some procedures use the SVD decomposition to estimate the rank of $\boldsymbol{W}_{\mathbf{1}} \boldsymbol{Z}_{\boldsymbol{f}} \boldsymbol{W}_{\mathbf{2}}$, which is the number of singular values statistically different from zero and coincides with the system order. In this line, Bauer (2001) proposes a criterion, called $S V C$ or "Singular Value Criterion", to determine the smaller non-null singular value. The criterion is defined as:

$$
S V C(n)=\hat{\sigma}_{n+1}^{2}+C(T) d(n)
$$

where $\hat{\sigma}_{n+1}$ is the $n+1$ singular value of $\boldsymbol{W}_{\mathbf{1}} \boldsymbol{Z}_{\boldsymbol{f}} \boldsymbol{W}_{\mathbf{2}}$ and $d(n)=2 n m$ denotes the number of parameters of a (1a-1b) system, excluding those in the error covariance matrix. Note that the singular values are denoted by $\sigma_{j} \forall j=1,2, \ldots, i m$ as the canonical correlations. This notation is motivated by the fact that, when Larimore-type method are used, the weighting matrices $\boldsymbol{W}_{\mathbf{1}}, \boldsymbol{W}_{\mathbf{2}}$ are such that the singular values of $\boldsymbol{W}_{1} \boldsymbol{Z}_{\boldsymbol{f}} \boldsymbol{W}_{\mathbf{2}}$ coincide with the canonical correlations between $\boldsymbol{Z}_{\boldsymbol{p}}$ and $\boldsymbol{Z}_{\boldsymbol{f}}$. In that case, the idea is close to that of Tsay (1989).

Equation (11) compares the information contained in $\hat{\sigma}_{n+1}$, with a penalty function weighted by the number of parameters in the model. The estimated order is obtained as the argument that minimizes (11). $C(T)$ must fulfill some requirements to assure the consistency of the method. Bauer (2001) shows that $S V C$ with $C(T)=\log T / T$ outperforms several information criteria when relatively large samples are analyzed, but recognizes that the penalty function is somewhat arbitrary. The author also finds that the choice of the weighting matrix 
$\boldsymbol{W}_{\mathbf{1}}$, used to solve the subspace reduced-rank regression, has a large impact on the performance of $S V C$ in finite samples.

\subsection{A posteriori methods}

Other useful tools to determine the system order are the, so-called, information criteria. The well-known $A I C$ (Akaike, 1976), SBC (Schwartz, 1978) or $H Q$ (Hannan and Quinn, 1979) belong to this family. A general representation of all these criteria is:

$$
I C(n)=\frac{-2 \log l(n)}{T}+C(T) d(n)
$$

where, $l(n)$ and $d(n)$ denote, respectively, the log-likelihood function value and the number of parameters corresponding to a system of order $n . C(T)$ is again a penalty function that depends on the criterion. In $A I C C(T)=2 / T$, in $S B C$ $C(T)=\log T / T$ and in $H Q, C(T)=2 \log \log T / T$.

These a posteriori procedures are ell known and widely employed, see recently Stoica et al. (2004) or Bengtsson and Cavanaugh (2006). In practice they have an important drawback, as they require estimating several models and, therefore, may be computationally expensive.

\section{Main results}

This section presents three criteria to determine the system order. Two of them are conceived to improve the performance of the $S V C$ in small samples, while maintaining its consistency. The third one is devised to be more robust than 
alternative methods. Finally, we describe a method to determine the regular and seasonal orders in series with seasonal fluctuations.

\subsection{Estimating the order of non-seasonal systems}

To improve the performance of $S V C$ we propose two enhancements: refining the choice of the weighting matrix and optimizing the penalty function through simulation.

The first improvement consists of substituting $\boldsymbol{W}_{\mathbf{1}}=\left(Z_{f} Z_{f}^{\prime}\right)^{-\frac{1}{2}}$, hereafter $\boldsymbol{\Omega}_{\mathbf{1}}$, by $\boldsymbol{W}_{1}=\left(\tilde{Z}_{f} \tilde{Z}_{f}^{\prime}\right)^{-\frac{1}{2}}$, from now on $\boldsymbol{\Omega}_{2}$, where $\tilde{Z}_{f}$ contains the residuals of regressing $\boldsymbol{Z}_{\boldsymbol{f}}$ onto $\boldsymbol{Z}_{\boldsymbol{p}}$ in a previous step. This choice is supported by the fact that $\boldsymbol{\Omega}_{\mathbf{2}}$ is the prediction error covariance matrix obtained from an autocorrelated noise term and therefore, as in generalized least squares, it should be an adequate weighting matrix. The choice of this weighting matrix will change the finite sample results of the procedure but its consistency will not be affected, provided that $\operatorname{rank}\left(\boldsymbol{\Omega}_{\mathbf{2}}\right) \geq n$.

The second enhancement consists of using a refined penalty function, denoted by $H(T, i)$, which depends not only on the sample size, but also on the dimension of the row space of the output BHM. Building on this idea, we define the criterion $N I D C$, which stands for " $n$ identification criterion", as:

$$
N I D C(n)=\hat{\sigma}_{n+1}^{2}+H(T, i) d(n)
$$

where, again, $d(n)=2 n m$ denotes the number of parameters. To specify $H(T, i)$ with good performance both, when $T \rightarrow \infty$ and also in finite samples, we build 
on the idea of Bengtsson and Cavanaugh (2006), who optimize the small sample performance of AIC-type criteria through Monte Carlo simulations.

In the following simulations, we will consider the data generating process (DGP) $z_{t}=a_{t}$, with $a_{t} \sim \operatorname{iidN}(0,1)$ and, accordingly, we will optimize the penalty criterion to check whether the DGP is white noise. These decisions are mitivated by the fact that the resulting criterion will be applied to a sequence of models, with increasing orders, until finding the minimum $n$ which corresponding model filters the data autocorrelation to white noise residuals. On the other hand, choosing a white noise DGP has a secondary advantage, as the properties needed by NIDC to assure a consistent order estimation are straightforward. In this case, NIDC will determine $\hat{n}=0$ instead of $\hat{n}=1$, if $N I D C(0)<N I D C(1)$, or substituting, if $\hat{\sigma}_{1}^{2}<\hat{\sigma}_{2}^{2}+2 H(T, i)$, since, in this particular case, $d(n)=2 n$. Rearranging terms we obtain, $\left(\hat{\sigma}_{1}^{2}-\hat{\sigma}_{2}^{2}\right) / 2<H(T, i)$. Likewise, to obtain $\hat{n}=0$ instead of $\hat{n}=2$, it must fulfill, $\left(\hat{\sigma}_{1}^{2}-\hat{\sigma}_{3}^{2}\right) / 4<H(T, i)$ and so on. Thus, we define $\nu_{j}$ as:

$$
\nu_{j}=\frac{\hat{\sigma}_{1}^{2}-\hat{\sigma}_{j+1}^{2}}{2 j}
$$

with $j=1, \ldots, i-1$. Therefore the optimal penalty term must be larger than $\nu_{j}$ assuring a correct performance of the criterion for this specific process.

\section{[INSERT FIGURE 1]}

Figure 1 shows that $H(T, i)=e^{-2} T^{-.9} i^{1.6}$ is an estimate of the lower bound below which one can find (by simulations) about the $95 \%$ of the realizations of $\nu_{1}$, a larger percentile of $\nu_{2}$ and in general of $\nu_{j}$ for $j=3, \ldots, i-1$. As we can see in the Figure, $C(T)=\log T / T$ is systematically above $H(T, i)$ in short samples, 
but the distance between them decreases when $T \rightarrow \infty$. This partially explains the different performance of both criteria in Section 5. Moreover, following the Proposition 4.1, the use of $H(T, i)$ in NIDC can be extended to other DGPs.

Proposition 4.1 The penalty function $H(T, i)=e^{-2} T^{-.9} i^{1.6}$, that fits $\nu_{1}$, assures the almost sure consistency of the system order estimated by minimizing NIDC(n).

The proof is given in Appendix A.

By construction, NIDC tends to overestimate $n$ in finite samples when compared to $S V C$, although both criteria lead to consistent estimation of the system order. Note that this is not a drawback since, as we will see in the simulations, $S V C$ shows a significant underestimate bias in short samples. This happens because $C(T)$ is systematically smaller than the 95 percentile of the realizations of $\nu_{1}$, see Figure 1. Moreover, provided that the sample size is reasonable, we think that overestimating is better than underestimating $n$, as further steps in modelbuilding may lead, through the pruning of insignificant parameters, to the correct dimension. In comparison, the standard diagnostics provided by estimation will not reveal the right dimension if the initial value is underestimated.

In any case, the performance of the different methods depends on the DGP and the sample size. This fact explains why there are so many procedures in the literature: none of them dominates systematically the rest and, as a consequence, the ability of each method to choose the right system order depends critically on the dynamics of the data. This lack of robustness motivates the idea of combining several methods, to avoid extreme (sometimes good, sometimes bad) performances. 
To this end, we suggest the following procedure:

1) Compute all (or a selection of) the criteria discussed in Sections 3 and 4: i) $S V C_{\Omega_{2}}$, which is the Bauer's original $S V C$ but computed with our weighting

matrix $\tilde{Z}_{f} \tilde{Z}_{\boldsymbol{f}}^{\prime}$, ii) the proposed $N I C D$, iii) $A I C, S B C$ and $H Q$, and iv) the $\chi^{2}$ test due to Tsay (1989). Despite the large number of criteria involved the computational cost is acceptable because, for each $n$, we only need to run a single least-squares subspace regression.

2) Set $\hat{n}$ as the value chosen by most methods, i.e., the sample mode. The mode is not necessarily unique, as different values of $\hat{n}$ can be chosen by the same number of methods. In this case, given our preference for overparametrization, we suggest picking the larger $\hat{n}$.

As we will see in Section 5, this Mode-based Criterion $(M b C)$ is robust and diversifies the risk of error when choosing the system order.

\subsection{Estimating the order in seasonal systems}

An important limitation of existing methods is that they cannot cope with multiplicative seasonal processes. To see this, consider the very common $\mathrm{MA}(1) \times$ $\mathrm{MA}(1)_{s}$ process, where the seasonal frequency of the data is $s=12$. In the best case, a standard order-determination method would choose $\hat{n}=13$. In comparison, a seasonality-tolerant method, such as that of Box and Jenkins (1976), would choose two different orders: one for the regular subsystem and another one for the seasonal subsystem. We will denote these orders by $n_{r}$ and $n_{s}$ respectively. 
Obviously, none of the previously revised methods could provide this double choice.

To solve this issue, we propose decomposing the process (1a-1b) into a seasonal subsystem:

$$
\begin{aligned}
x_{t+s}^{s} & =\Phi_{s} x_{t}^{s}+E_{s} r_{t} \\
z_{t} & =H_{s} x_{t}^{s}+r_{t}
\end{aligned}
$$

and a regular subsystem:

$$
\begin{aligned}
x_{t+1}^{r} & =\Phi_{r} x_{t}^{r}+E_{r} \psi_{t} \\
r_{t} & =H_{r} x_{t}^{r}+\psi_{t}
\end{aligned}
$$

and applying the methods previously described to determine the order of each subsystem. Note that these processes are defined in different frequencies, as $\boldsymbol{x}_{\boldsymbol{t}}^{\boldsymbol{s}}$ propagates in increments of $s$ periods in (14a), while $\boldsymbol{x}_{\boldsymbol{t}}^{\boldsymbol{r}}$ propagates in increments of one period in (15a); $\boldsymbol{r}_{\boldsymbol{t}}$ is an unobserved input of the seasonal subsystem (14a-14b) representing the regular structure of the original process. To estimate sequentially both subsystems, we assume that $\boldsymbol{r}_{\boldsymbol{t}}$ is a white noise term in the $s$ frequency, uncorrelated with $\boldsymbol{x}_{\boldsymbol{t}}^{\boldsymbol{s}} \forall t$. To support this assumption, Appendix B shows that the expectation of $\boldsymbol{r}_{\boldsymbol{t}+\boldsymbol{j}} \boldsymbol{r}_{\boldsymbol{t}}^{\prime}$ for all $j=s, 2 s, \ldots$ converges to zero at a speed governed by the seasonal period $s$ and the eigenvalues of $\boldsymbol{\Phi}_{\boldsymbol{r}}$.

This approximation, which is exact when the regular system follows an $\operatorname{MA}(q)$ with $q<s$, allows us to estimate both subsystems using subspace methods. How- 
ever, in (14a-14b) the states propagate in increments of $s$ periods and, as a consequence, we cannot use the standard BHM defined in (5). This issue can be solved by defining the Seasonal Block Hankel Matrices (SBHM) of period $s$, as:

$$
\boldsymbol{Z}_{\boldsymbol{p}}^{\boldsymbol{s}}=\left(\begin{array}{ccc}
z_{1} & \ldots & z_{T-s(2 i-1)} \\
z_{s+1} & \ldots & z_{T-s(2 i-2)} \\
\vdots & & \vdots \\
z_{s(i-1)+1} & \ldots & z_{T-s i}
\end{array}\right) ; \quad \boldsymbol{Z}_{\boldsymbol{f}}^{\boldsymbol{s}}=\left(\begin{array}{ccc}
z_{s i+1} & \ldots & z_{T-s(i-1)} \\
z_{s(i+1)+1} & \ldots & z_{T-s(i-2)} \\
\vdots & & \vdots \\
z_{s(2 i-1)+1} & \ldots & z_{T}
\end{array}\right)
$$

Note that these matrices are analogous to the standard ones, although the time indices in each row are adapted to the seasonal period. Similarly, we denote both, the past and future-seasonal information blocks by $\boldsymbol{Z}_{\boldsymbol{p}}^{\boldsymbol{s}}$ and $\boldsymbol{Z}_{\boldsymbol{f}}^{\boldsymbol{s}}$ respectively. Building on these matrices, one can estimate the seasonal parameters and, accordingly, the order of the seasonal subsystem, $n_{s}$, by applying any subspace method to the seasonal regression model:

$$
Z_{f}^{s}=O_{i}^{s} M^{s} Z_{p}^{s}+V_{i}^{s} \Psi_{f}^{s}
$$

instead of the standard subspace regression (8).

This generalization of conventional subspace methods allows us to apply the procedures discussed in Sections 3 and 4.1 to determine the seasonal and regular orders, by means of the following structured method:

1) Create the SBHM and compute the seasonal order $\hat{n}_{s}$ by applying the procedures previously described to the seasonal subsystem (14a-14b). 
2) Estimate the seasonal subsystem, conditional to the choice done in step 1), using e.g., the techniques shown in Section 2. Compute the residuals of this subsystem, $\hat{r}_{t}$.

3) Determine the order of the regular subsystem, $\hat{n}_{r}$, by applying standard methods to $\hat{r}_{t}$.

Note that if SVD methods $\left(S V C_{\Omega_{1}}, S V C_{\Omega_{2}}\right.$ or $\left.N I D C\right)$ are applied to determine the system orders, the seasonal subsystem (14a-14b) does not need to be estimated, as $\hat{n}_{s}$ can be directly obtained from $\boldsymbol{W}_{\mathbf{1}} \boldsymbol{Z}_{\boldsymbol{f}}^{\boldsymbol{s}} \boldsymbol{W}_{\mathbf{2}}$, where $\boldsymbol{W}_{\mathbf{1}}$ and $\boldsymbol{W}_{\mathbf{2}}$ are as in Section 2 but replacing $\boldsymbol{Z}_{\boldsymbol{p}}$ by $\boldsymbol{Z}_{\boldsymbol{p}}^{\boldsymbol{s}}$ and $\boldsymbol{Z}_{\boldsymbol{f}}$ by $\boldsymbol{Z}_{\boldsymbol{f}}^{\boldsymbol{s}}$.

The order of steps 1) to 3) in the previous procedure could be reversed, to determine first $\hat{n}_{r}$ and, afterwards, $\hat{n}_{s}$. However this is not advisable because: i) in series with a short seasonal period, e.g. quarterly data with $s=4$, it would be difficult to determine the correct regular order in the first step, and ii) the choice of $\hat{n}_{r}$ is often more complex than that of $\hat{n}_{s}$, increasing the chance to contaminate the second decision with the effects of a previous specification error.

\section{Monte Carlo results}

This section analyzes the performance of several criteria by simulating univariate and multivariate models. Tables 1 to 8 show the percentage of replications where each criterion chose $n$ and the contiguous dimensions, $n-1$ and $n+1$ in nonseasonal processes, while Tables 9 and 10 offer the relative frequency of hits in two seasonal models. In all cases, we discard the first 50 draws of each replication 
to improve randomization. Each table shows the results obtained using $S V C_{\Omega_{2}}$, $N I C D, A I C, S B C, H Q$, the $\chi^{2}$ test and $M b C$. Additionally, $S V C_{\Omega_{1}}$, as presented in Bauer (2001), is included.

The specific formulations employed in this exercise have been chosen to show how any individual criterion may perform both, very well and badly, depending on the dynamic structure of the DGP and the sample size.

On the other hand, the univariate GDPs assumed in the first five tables show a sequence of common nonseasonal ARMA models, with increasingly complex dynamic structures. Accordingly, the white noise DGP in Table 1 is followed by an $\mathrm{AR}(1)$ process with low persistence, a strong $\mathrm{MA}(1)$ structure, an $\mathrm{AR}(2)$ with complex roots in Table 4 and, finally, an $\operatorname{ARMA}(2,1)$ in Table 5. These basic results are supplemented by the three vector processes assumed in tables 6,7 and 8 , being the last two of them taken from the literature. The last two processes were chosen to illustrate the performance of the proposed decomposition for seasonal processes. The model in Table 9 adds a seasonal MA(1) term to the $\mathrm{AR}(2)$ with complex roots that was previously assumed and, finally, the model in Table 10 is a somewhat artificial combination of different seasonal factors, that we specified to show how our decomposition procedure is able to cope with multiple periods.

Table 1 shows that the best estimates are provided by $S B C$. In this case there is no risk of underestimation, as the DGP is a white noise, so $S V C_{\Omega_{1}}$ dominates our two alternative criteria which, in small samples tend to overestimate $n$. 
The second model considered is an autoregressive process with small persistence. This structure deteriorates the performance of all the methods. In this case: i) $A I C$ displays the best performance for almost every sample size, ii) $S V C_{\Omega_{2}}$ and NIDC widely dominate $S V C_{\Omega_{1}}$ and iii) assuming that a small overparametrization can lead to an adequate model in next steps, NIDC shows remarkable results in very small samples. The DGP M3 has also $n=1$, but the moving average parameter is large enough to improve the outcomes of all the methods. Again $S B C$ exhibits the best behavior, reaching the asymptotic value with a moderate sample size.

Table 4 displays the results obtained with an autoregressive model with complex roots. This time NIDC beats the rest of the methods for almost all the samples analyzed. Surprisingly, $S B C$ presents the worst results, with less than $10 \%$ of correct estimates even with a large sample.

The DGP M5 is an $\operatorname{ARMA}(2,1)$ model with complex roots, so its order is again 2. Including a moving average term improves substantially the results provided by all the methods. Again, NIDC and AIC show the best behavior in small and large samples, respectively.

Table 6 summarizes the results obtained with a bivariate $\operatorname{VARMA}(1,1)$ process, with $n=2$. One of the series has an autoregressive term close to be cancelled out by a moving average root. The performance, which is good in all cases for large samples, is very heterogeneous in small samples. $S V C_{\Omega_{1}}$ tends to underestimate $n$, while this is corrected in $S V C_{\Omega_{2}}$ and $N I D C$ that clearly dominate it when 
$T \leq 100$

Tables 7 and 8 display the results corresponding to two trivariate $\operatorname{VARMA}(1,1)$ models, with $n=3$. In both cases, we omit the smaller sample sizes due to the complexity of the systems. The first one, M7, is taken from Koreisha and Pukkila (1989). The results obtained are similar to those of previous cases, but the differences in performance are amplified by the increased complexity of the order selection problem. $S V C_{\Omega_{1}}$ shows a clear bias towards underestimation and does not find any $\hat{n}=n-1$ even when $T \leq 100$. Its enhanced versions produce much better results, as $N I D C$ and $S V C_{\Omega_{2}}$ show a reasonably good behavior. Note, also, that $M b C$ presents remarkably good results. The second process, M8, is taken from Reinsel et al. (1992). The results are better than those of Table 7, because the structure of M7 is closer to a lower-order representation. In this case the $\chi^{2}$ test beats all the criteria when $T \leq 100$, and all the methods behave well when the sample is large.

Tables 9 and 10 present two seasonal models. Here we are interested in assessing their performance when combined with the method described in Section 4.2. In both models the sample sizes are increased to represent the typical size of a seasonal data sample. The orders are estimated by following the stages stated in Section 4.2, using $M b C$ to obtain the residuals in step 2).

M9 adds to M4 a monthly MA(1) term. In this case all the methods compared behave well, although the $\chi^{2}$ test has some difficulties when $T=100$. In the second step, the results are similar to those in Table 4 . This suggests that the 
method employed to determine the seasonal order does not distort the estimation of the regular order.

Model M10 has multiple seasonal factors at frequencies 3, 9 and 36. This could happen, for instance, with financial data observed once each 10 days. Table 10 reveals: i) the remarkable performance of $S B C$ in these models, and ii) the outstanding ability our decomposition to estimate the seasonal order in each frequency, in combination with all the criteria considered. Note that, when using $a$ posteriori criteria, we have estimated only 12 models as we have tried $n_{s}=0,1,2$ for each factor. On the other hand, if we search the dynamic of this data using the same methods over the currently BHM, we should estimate, at least, 50 models as 49 is the order of the original process.

Previous results support the consistency of all the methods compared and also provide specific clues about their specific performance.

Criteria based on $S V D$. In small samples the performance of $S V C_{\Omega_{1}}$ is worst than that of $S V C_{\Omega_{2}}$ and NIDC. This difference is even more remarkable in multivariate processes. Note also that NIDC is often the best method when the persistence is small.

Information Criteria. The information criteria considered display different performances. $A I C$ occasionally overestimates $n$ in univariate process but provides good enough results with multivariate DGPs (see Gonzalo and Pitarakis, 2002). $S B C$ is one of the best options in both, univariate and multivariate processes, 
when the sample is large and/or the parameters persistent enough. Nevertheless, it shows the worst performance with low-persistence DGPs. $H Q$ displays an intermediate performance: i) better than $S B C$ (but worst than $A I C$ ) in low-persistence AR processes or when there are AR and MA factors close to cancellation, but ii) worst than $S B C$ (better than $A I C$ ) in other cases.

$\chi^{2}$ test. The $\chi^{2}$ test shows poor results in small samples, improving substantially as the sample size grows. In univariate process it is not the best option while in multivariate systems its performance improves remarkably.

Mode-based criterion. This procedure is very robust, as it is never the worst option, but sometimes is the best (see Table $7, T \geq 300$ ). In our opinion, this should be the method-of-choice for automatic implementations useful, e.g., when the analyst has to work with a large set of series.

\section{Conclusions}

This paper discusses how can one estimate the order of a SS process using subspace methods. These methods are powerful, flexible and computationally efficient.

Our first contribution consists of proposing two new criteria that improve the small-sample performance of Bauer (2001) SVC method. Second, we propose an automatic model-building procedure based on the sample mode of several methods. A simulation analysis confirms that the mode-based procedure is more robust than any specific criterion. Last, we extend the use of any criterion to processes 
with seasonality. Monte Carlo results show that the resulting criteria display a remarkable performance, even in a complex multiple seasonality framework.

In comparison with the popular VAR approach, which is very close to ours in terms of simplicity and computational efficiency, the methodology illustrated here has pros and cons. On the minus side, the rank-deficient GLS regressions required by subspace methods are more complex than OLS or GLS VAR. However it has two clear advantages over VAR, as it: i) supports seasonal time series and ii) is intrinsically more parsimonious, as it looks for the right system order by increasing the dimension of the state vector one-by-one. To clarify this remark, consider e.g., a vector of $m$ time series and the decision to increase the order of a $\operatorname{VAR}(p)$ model to $\operatorname{VAR}(p+1)$. To do so implies increasing the dimension of the state vector by adding $m$ new states, while our method would try all the possible choices in the sequence of $1,2, m$ additional states.

The algorithms described in this article are implemented in a MATLAB toolbox for time series modeling called $E^{4}$. The source code of this toolbox is freely provided under the terms of the GNU General Public License and can be downloaded at www.ucm.es/info/icae/e4. This site also provides a complete user manual for the Toolbox and other reference materials. 


\section{References}

Akaike, H. (1976). Canonical Correlation Analysis of Time Series and the Use of an Information Criterion. Academic Press.

Aoki, M. (1990). State Space Modelling of Time Series. Springer Verlag, New York.

Bauer, D. (2001). Order estimation for subspace methods. Automatica, 37:15611573.

Bengtsson, T. and Cavanaugh, J. (2006). An improved akaike information criterion for state-space model selection. Computational Statistics and Data Analysis, 50(10):2635-2654.

Box, G. E. P. and Jenkins, G. M. (1976). Time Series Analysis, Forecasting and Control. Holden-Day, San Francisco, 2nd ed. edition.

Casals, J., Sotoca, S., and Jerez, M. (1999). A fast stable method to compute the likelihood of time invariant state space models. Economics Letters, 65(3):329337.

Chui, C. K. and Chen, G. (1999). Kalman Filtering with Real-Time Applications. Berlin, Springer.

Cooper, D. M. and Wood, E. F. (1982). Identifying multivariate time series models. Journal of Time Series Analysis, 3:277-293.

Eckart, C. and Young, G. (1936). The approximation of one matrix by another of lower rank. Psychometrika, 1:211-218.

Gonzalo, J. and Pitarakis, J. Y. (2002). Lag length estimation in large dimensional systems. Journal or Time Series Analysis, 23:401-423.

Hannan, E. J. and Quinn, B. G. (1979). The determination of the order of an autoregression. Journal of the Royal Statistical Association, B Series, 41:713723.

Katayama, T. (2005). Subspace Methods for System Identification. Springer Verlag.

Koreisha, S. G. and Pukkila, T. H. (1989). Fast linear estimation methods for vector autoregressive moving average models. Journal of Time Series Analysis, 10:325-339. 
Larimore, W. E. (1983). System identification, reduced-order filtering and modeling via canonical variate analysis. Proceedings of the American Control Conference, ACC, San Francisco, pages 445-451.

Larimore, W. E. (1990). Canonical variate analysis in identification, filtering and adaptive control. Proceedings of the 29th Conference on Decision and Control, Hawaii, pages 596-604.

Peternell, K., Scherrer, W., and Deistler, M. (1996). Statistical analysis of novel subspace identification methods. Signal Processing, 52:161-177.

Reinsel, G. C., Basu, S., and Yap, S. F. (1992). Maximum likelihood estimators in the multivariate autoregressive moving average model from a generalized least squares viewpoint. Journal of Time Series Analysis, 13(2):133-145.

Schwartz, G. (1978). Estimating the dimension of a model. The Annals of Statistics, 6:1-48.

Sorelius, J. (1999). Subspace-Based Parameter Estimation Problems in Signal Processing. PhD thesis, Uppsala University.

Stoica, P., Selen, Y., and Jian Li (2004). On information criteria and the generalized likelihood ratio test of model order selection. Signal Processing Letters, IEEE, 11(10):794-797.

Stoica, P. and Söderström, T. (1982). On nonsingular information matrices and local identifiability. International Journal Control, 36:323-329.

Tiao, G. C. and Tsay, R. S. (1989). Model specification in multivariate time series. Journal of the Royal Statistical Society, B Series, 51(2):157-213.

Toscano, E. M. M. and Reisen, V. A. (2000). The use of canonical correlation analysis to identify the order of multivariate arma models: Simulation and application. Journal of Forecasting, 19:441-455.

Tsay, R. S. (1989). Identifying multivariate time series models. Journal of Time Series Analysis, 10(4):357-372.

Viberg, M. (1995). Subspace-based methods for the identification of the linear time-invariant systems. Automatica, 31(12):1835-1852.

Wei, W. W. S. (1990). Time Series Analysis. Addison Wesley.

Woodside, C. M. (1971). Estimation of the order of linear systems. Automatica, $7: 727-733$. 


\section{Appendix A}

Proof. The conditions on the penalty function that are sufficient for the almost sure consistency of the order estimated by minimizing (13) are: 1) $H(T, i) T>0$, 2) $H(T, i) \rightarrow 0$ and, 3) $H(T, i) T /\left(i^{2} \log \log T\right) \rightarrow \infty$, when $T \rightarrow \infty$ (see Bauer, 2001, Theorem 3).

Although in practice we implement $i=\max \left(4, h_{t}\right)$, being $h_{t}$ the integer closer to $\log T$, in sections 2) and 3) of this proof we consider, without lost of generality, that $i=\log T$. Let $H(T, i)=a T^{-b} i^{c}$ with $a=e^{-2}, b=.9$ and $c=1.6$, under these conditions:

1) It is straightforward to see that,

$$
H(T, i) T=a T^{-b+1} i^{c}>0, \quad \forall T>0
$$

2) The limit,

$$
\lim _{T \rightarrow \infty} a T^{-b}(\log T)^{c}=\frac{a(\log T)^{c}}{T^{b}}
$$

converges to the indeterminate $\frac{\infty}{\infty}$. Applying twice L'Hôpital rule we obtain,

$$
\lim _{T \rightarrow \infty} \frac{a c(c-1)(\log T)^{c-2}}{b^{2} T^{b}}=0
$$

and as the numerator tends to zero and the denominator to infinity, we get:

$$
\lim _{T \rightarrow \infty} H(T, i)=0
$$


3) Finally,

$$
\frac{H(T, i) T}{i^{2} \log \log T}=\frac{a T^{-b+1}(\log T)^{c-2}}{\log \log T}
$$

where the numerator can be written as,

$$
a T^{-b+1}(\log T)^{c-2}=\frac{a(\log T)^{c-2}}{T^{b-1}}
$$

that gives the indeterminate $\frac{0}{0}$. By L'Hôpital we get,

$$
\lim _{T \rightarrow \infty} \frac{a(-b+1) T^{-b+3}}{(-c+2)(\log T)^{-c+1}}=\infty
$$

and therefore both, the numerator and the denominator, tend to infinity. We can then apply L'Hôpital to the initial limit, obtaining:

$$
\lim _{T \rightarrow \infty} \frac{a T^{-b+1}(\log T)^{c-2}}{\log \log T}=\lim _{T \rightarrow \infty} a T^{1-b}\left[(1-b)(\log T)^{c-1}+(c-2)(\log T)^{c-2}\right]=\infty
$$

\section{Appendix B}

By recursive substitution in (15a) and shifting time subscripts, the regular subsystem (15a-15b) can be expressed as:

$$
\begin{aligned}
x_{t+s}^{r} & =\Phi_{r}^{s-1} x_{t+\mathbf{1}}^{r}+\sum_{j=0}^{s} \Phi_{r}^{j} E_{r} \psi_{t+s-j-1} \\
r_{t+s} & =H_{r} x_{t+s}^{r}+\psi_{t+s}
\end{aligned}
$$


From these two equations, it is easy to see that:

$$
\mathrm{E}\left[\boldsymbol{r}_{\boldsymbol{t}+\boldsymbol{s}} \boldsymbol{r}_{\boldsymbol{t}}^{\prime}\right]=\mathrm{E}\left[\left(\boldsymbol{H}_{\boldsymbol{r}} \boldsymbol{x}_{\boldsymbol{t}+\boldsymbol{s}}^{\boldsymbol{r}}+\boldsymbol{\psi}_{\boldsymbol{t}+\boldsymbol{s}}\right) \boldsymbol{r}_{\boldsymbol{t}}^{\prime}\right]=\boldsymbol{H}_{\boldsymbol{r}} \boldsymbol{\Phi}_{\boldsymbol{r}}^{\boldsymbol{s}-1} \mathrm{E}\left[\boldsymbol{x}_{\boldsymbol{t}+1}^{\boldsymbol{r}} \boldsymbol{r}_{\boldsymbol{t}}^{\prime}\right]
$$

where $\mathrm{E}[\cdot]$ is the mathematical expectation. Since $\boldsymbol{z}_{\boldsymbol{t}}$ is stationary, and as a consequence all the eigenvalues of $\boldsymbol{\Phi}_{\boldsymbol{r}}$ are inside the unit circle, then, provided that $s$ is large enough, we will assume that:

$$
\mathrm{E}\left[\boldsymbol{r}_{\boldsymbol{t}+\boldsymbol{j}} \boldsymbol{r}_{\boldsymbol{t}}^{\prime}\right] \simeq \mathbf{0}, \quad \forall j=s, 2 s, \ldots
$$




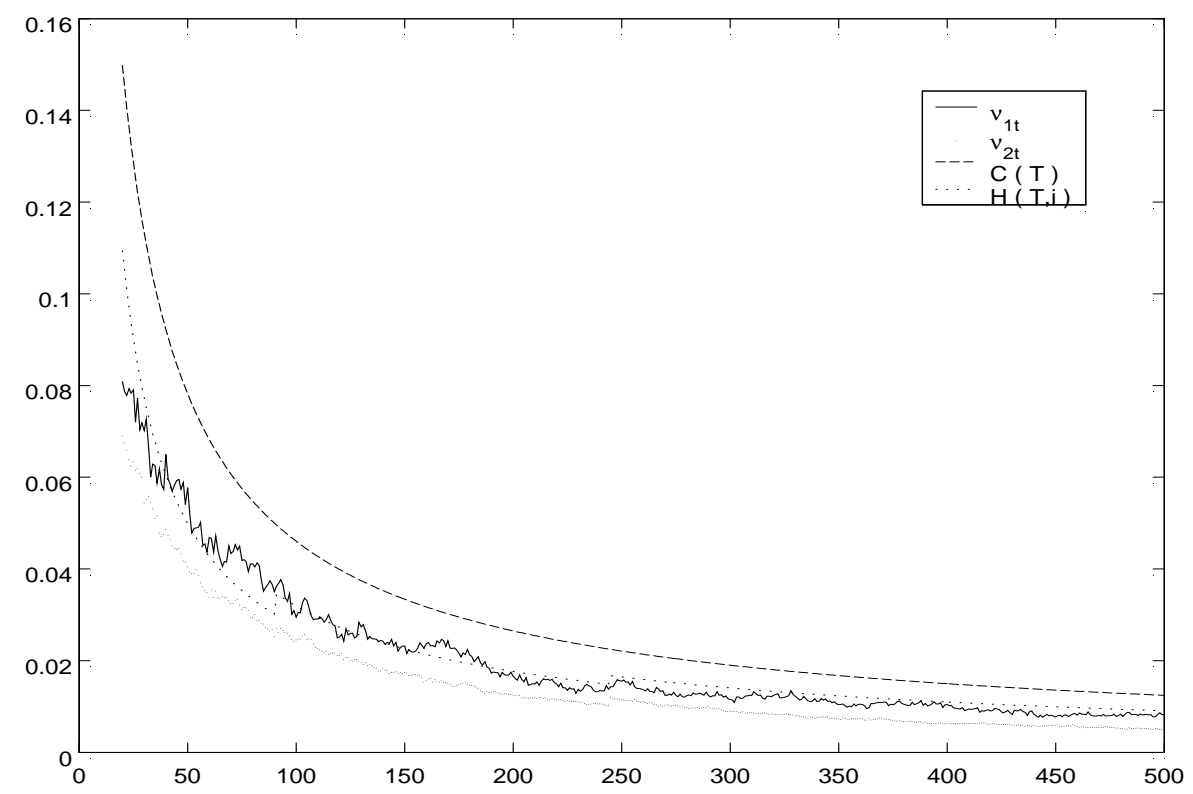

Figure 1: Estimated penalty function of NIDC. $\nu_{1}$ and $\nu_{2}$ are computed using the singular values obtained as the 95 percentile of 2000 simulations of the univariate model $z_{t}=a_{t} . \mathrm{H}(\mathrm{T}, \mathrm{i})$ is the lost function fitted to $\nu_{1}$, proposed in NIDC. C $(T)$ is the penalty term suggested in Bauer (2001). 
Table 1: System order estimates, M1 model *

$\operatorname{M1}(n=0): z_{t}=a_{t}, \quad a_{t} \sim \operatorname{iidN}(0,1)$.

\begin{tabular}{|c|c|c|c|c|c|c|c|c|}
\hline $\mathrm{T}$ & $S V C_{\Omega_{1}}$ & $S V C_{\Omega_{2}}$ & $N I D C$ & $A I C$ & $S B C$ & $H Q$ & $\chi^{2}$ & $M b C$ \\
\hline \multicolumn{9}{|c|}{$\hat{n}=n$} \\
\hline 30 & 0.945 & 0.812 & 0.703 & 0.828 & 0.965 & 0.846 & 0.948 & 0.899 \\
\hline 50 & 0.948 & 0.850 & 0.726 & 0.816 & $\overline{0.979}$ & 0.889 & & 0.919 \\
\hline 100 & 0.918 & 0.862 & 0.797 & 0.768 & $\underline{0.975}$ & 0.908 & 0.955 & 0.927 \\
\hline 300 & 0.905 & 0.889 & 0.867 & 0.722 & $\overline{0.990}$ & 0.944 & 0.9 & 0.948 \\
\hline 500 & 0.934 & 0.919 & 0.892 & 0.711 & 0.993 & 0.947 & 0.950 & 0.953 \\
\hline \multicolumn{9}{|c|}{$\hat{n}=n+1$} \\
\hline 30 & 0.053 & 0.152 & 0.250 & 0.156 & 0.035 & 0.141 & & 0.098 \\
\hline 50 & 0.050 & 0.132 & 0.266 & 0.172 & 0.020 & 0.115 & 0.0 & 0.065 \\
\hline 100 & 0.075 & 0.118 & 0.184 & 0.203 & 0.025 & 0.092 & 0.036 & 0.057 \\
\hline 300 & 0.083 & 0.096 & 0.110 & 0.239 & 0.010 & 0.055 & 0.042 & 0.041 \\
\hline 500 & 0.059 & 0.071 & 0.092 & 0.239 & 0.007 & 0.063 & 0.034 & 0.031 \\
\hline
\end{tabular}

* The table shows the percentage of cases where each criteria chose $n$, and the contiguous choice $n+1$. The number of replications is 2000. The methods analyzed are the following: $S V C_{\Omega_{1}}$ from Bauer (2001), $S V C_{\Omega_{2}}$ that has the same structure but computed with the proposed weighting matrix $\Omega_{2}=\left(\tilde{Z}_{f} \tilde{Z}_{f}^{\prime}\right)^{-\frac{1}{2}}, N I D C$ which is as $S V C_{\Omega_{2}}$ but replacing the penalty term $C(T)=\log T / T$ by $H(T, i)=e^{-2} T^{-.9} i^{1.6} . A I C, S B C$ and $H Q$ are the information criteria due to Akaike (1976), Schwartz (1978) and Hannan and Quinn (1979) respectively. $\chi^{2}$ is the test proposed by Tsay (1989) with conventional significance level $5 \%$ and $M b C$ is the $\hat{n}$ value most often for each realization in the criteria collection, except $S V C_{\Omega_{1}}$. For each sample size, the largest relative frequency of hits is underlined. 
Table 2: System order estimates, M2 model .

$\operatorname{M} 2(n=1):(1-0.2 B) z_{t}=a_{t}, \quad a_{t} \sim \operatorname{iidN}(0,1)$.

\begin{tabular}{|c|c|c|c|c|c|c|c|c|}
\hline $\mathrm{T}$ & $S V C_{\Omega_{1}}$ & $S V C_{\Omega_{2}}$ & $N I D C$ & $A I C$ & $S B C$ & $H Q$ & $\chi^{2}$ & $M b C$ \\
\hline \multicolumn{9}{|c|}{$\hat{\bar{n}}=n-1$} \\
\hline 30 & 0.913 & 0.801 & 0.384 & 0.680 & 0.866 & 0.682 & 0.878 & 0.721 \\
\hline 50 & 0.861 & 0.728 & 0.445 & 0.551 & 0.818 & 0.672 & 0.841 & 0.657 \\
\hline 100 & 0.782 & 0.676 & 0.498 & 0.314 & 0.697 & 0.521 & 0.729 & 0.532 \\
\hline 300 & 0.486 & 0.415 & 0.373 & 0.037 & 0.273 & 0.130 & 0.246 & 0.212 \\
\hline 500 & 0.243 & 0.204 & 0.166 & 0.007 & 0.081 & 0.027 & 0.051 & 0.078 \\
\hline \multicolumn{9}{|c|}{$\hat{n}=n$} \\
\hline 30 & 0.086 & 0.185 & $\underline{0.385}$ & 0.315 & 0.134 & 0.305 & 0.098 & 0.272 \\
\hline 50 & 0.133 & 0.244 & 0.395 & $\underline{0.435}$ & 0.182 & 0.311 & 0.127 & 0.324 \\
\hline 100 & 0.204 & 0.290 & 0.408 & $\underline{0.649}$ & 0.301 & 0.470 & 0.238 & 0.446 \\
\hline 300 & 0.490 & 0.548 & 0.575 & $\underline{0.916}$ & 0.724 & 0.849 & 0.713 & 0.771 \\
\hline 500 & 0.724 & 0.757 & 0.778 & $\underline{0.967}$ & 0.915 & 0.961 & 0.906 & 0.922 \\
\hline \multicolumn{9}{|c|}{$\hat{n}=n+1$} \\
\hline 30 & 0.001 & 0.014 & 0.211 & 0.005 & 0.0 & 0.013 & 0.019 & 0.016 \\
\hline 50 & 0.006 & 0.027 & 0.115 & 0.013 & 0.0 & 0.017 & 0.026 & 0.019 \\
\hline 100 & 0.013 & 0.033 & 0.081 & 0.036 & 0.002 & 0.009 & 0.023 & 0.019 \\
\hline 300 & 0.020 & 0.036 & 0.047 & 0.043 & 0.003 & 0.021 & 0.027 & 0.015 \\
\hline 500 & 0.032 & 0.039 & 0.054 & 0.024 & 0.004 & 0.012 & 0.029 & 0.025 \\
\hline
\end{tabular}

* The table shows the percentage cases where each criteria chose $n$, and the contiguous choices $n-1$ and $n+1$. The number of replications is 2000. The methods analyzed are the following: $S V C_{\Omega_{1}}$ from Bauer (2001), $S V C_{\Omega_{2}}$ that has the same structure but computed with the proposed weighting matrix $\Omega_{2}=\left(\tilde{Z}_{f} \tilde{Z}_{f}^{\prime}\right)^{-\frac{1}{2}}, N I D C$ which is as $S V C_{\Omega_{2}}$ but replacing the penalty term $C(T)=\log T / T$ by $H(T, i)=e^{-2} T^{-.9} i^{1.6} . A I C, S B C$ and $H Q$ are the information criteria due to Akaike (1976), Schwartz (1978) and Hannan and Quinn (1979) respectively. $\chi^{2}$ is the test proposed by Tsay (1989) with conventional significance level $5 \%$ and $M b C$ is the $\hat{n}$ value most often for each realization in the criteria collection, except $S V C_{\Omega_{1}}$. For each sample size, the largest relative frequency of hits is underlined. 
Table 3: System order estimates, M3 model ${ }^{\star}$.

$\operatorname{M3}(n=1): z_{t}=(1-0.8 B) a_{t}, \quad a_{t} \sim \operatorname{iidN}(0,1)$.

\begin{tabular}{|c|c|c|c|c|c|c|c|c|}
\hline $\mathrm{T}$ & $S V C_{\Omega_{1}}$ & $S V C_{\Omega_{2}}$ & NIDC & $A I C$ & $S B C$ & $H Q$ & $\chi^{2}$ & $M b C$ \\
\hline \multicolumn{9}{|c|}{$\bar{c} \hat{n}=n-1$} \\
\hline 30 & 0.179 & 0.032 & 0.021 & 0.021 & 0.057 & 0.025 & 0.284 & 0.020 \\
\hline 50 & 0.019 & 0.001 & 0.0 & 0.0 & 0.006 & 0.0 & 0.018 & 0.0 \\
\hline 100 & 0.0 & 0.0 & 0.0 & 0.0 & 0.0 & 0.0 & 0.0 & 0.0 \\
\hline 300 & 0.0 & 0.0 & 0.0 & 0.0 & 0.0 & 0.0 & 0.0 & 0.0 \\
\hline 500 & 0.0 & 0.0 & 0.0 & 0.0 & 0.0 & 0.0 & 0.0 & 0.0 \\
\hline \multicolumn{9}{|c|}{$\hat{n}=n$} \\
\hline 30 & 0.793 & 0.819 & 0.761 & 0.932 & 0.934 & 0.929 & 0.676 & 0.919 \\
\hline 50 & 0.929 & 0.850 & 0.767 & 0.932 & 0.988 & 0.978 & 0.946 & 0.960 \\
\hline 100 & 0.941 & 0.907 & 0.813 & 0.935 & $\overline{0.998}$ & 0.981 & 0.965 & 0.967 \\
\hline 300 & 0.949 & 0.934 & 0.918 & 0.915 & $\overline{1.0}$ & 0.984 & 0.971 & 0.973 \\
\hline 500 & 0.954 & 0.944 & 0.931 & 0.909 & $\overline{1.0}$ & 0.981 & 0.966 & 0.980 \\
\hline \multicolumn{9}{|c|}{$\hat{n}=n+1$} \\
\hline 30 & 0.027 & 0.124 & 0.166 & 0.045 & 0.009 & 0.041 & 0.03 & 0.055 \\
\hline 50 & 0.048 & 0.120 & 0.171 & 0.067 & 0.006 & 0.022 & 0.017 & 0.029 \\
\hline 100 & 0.052 & 0.076 & 0.126 & 0.063 & 0.002 & 0.019 & 0.012 & 0.034 \\
\hline 300 & 0.044 & 0.053 & 0.062 & 0.082 & 0.0 & 0.016 & 0.013 & 0.019 \\
\hline 500 & 0.040 & 0.047 & 0.053 & 0.090 & 0.0 & 0.019 & 0.01 & 0.014 \\
\hline
\end{tabular}

* The table shows the percentage cases where each criteria chose $n$, and the contiguous choices $n-1$ and $n+1$. The number of replications is 2000. The methods analyzed are the following: $S V C_{\Omega_{1}}$ from Bauer (2001), $S V C_{\Omega_{2}}$ that has the same structure but computed with the proposed weighting matrix $\Omega_{2}=\left(\tilde{Z}_{f} \tilde{Z}_{f}^{\prime}\right)^{-\frac{1}{2}}, N I D C$ which is as $S V C_{\Omega_{2}}$ but replacing the penalty term $C(T)=\log T / T$ by $H(T, i)=e^{-2} T^{-.9} i^{1.6} . A I C, S B C$ and $H Q$ are the information criteria due to Akaike (1976), Schwartz (1978) and Hannan and Quinn (1979) respectively. $\chi^{2}$ is the test proposed by Tsay (1989) with conventional significance level $5 \%$ and $M b C$ is the $\hat{n}$ value most often for each realization in the criteria collection, except $S V C_{\Omega_{1}}$. For each sample size, the largest relative frequency of hits is underlined. 
Table 4: System order estimates, ${ }_{4} 4$ model $^{\star}$.

$\operatorname{M} 4(n=2):\left(1-0.4 B+0.3 B^{2}\right) z_{t}=a_{t}, \quad a_{t} \sim \operatorname{iidN}(0,1)$.

\begin{tabular}{|c|c|c|c|c|c|c|c|c|}
\hline $\mathrm{T}$ & $S V C_{\Omega_{1}}$ & $S V C_{\Omega_{2}}$ & $N I D C$ & $A I C$ & $S B C$ & $H Q$ & $\chi^{2}$ & $M b C$ \\
\hline \multicolumn{9}{|c|}{ 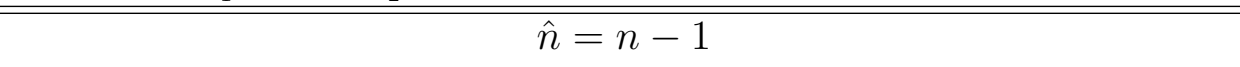 } \\
\hline 30 & 0.247 & 0.419 & 0.415 & 0.652 & 0.441 & 0.628 & 0.257 & 0.563 \\
\hline 50 & 0.439 & 0.555 & 0.480 & 0.800 & 0.673 & 0.803 & 0.520 & 0.664 \\
\hline 100 & 0.653 & 0.640 & 0.517 & 0.814 & 0.939 & 0.909 & 0.767 & 0.756 \\
\hline 300 & 0.487 & 0.445 & 0.401 & 0.460 & 0.958 & 0.796 & 0.464 & 0.527 \\
\hline 500 & 0.290 & 0.263 & 0.233 & 0.229 & 0.908 & 0.605 & 0.204 & 0.249 \\
\hline \multicolumn{9}{|c|}{$\hat{n}=n$} \\
\hline 30 & 0.026 & 0.147 & $\underline{0.326}$ & 0.085 & 0.022 & 0.062 & 0.082 & 0.110 \\
\hline 50 & 0.079 & 0.179 & $\overline{0.376}$ & 0.126 & 0.018 & 0.049 & 0.111 & 0.167 \\
\hline 100 & 0.199 & 0.283 & $\overline{0.402}$ & 0.182 & 0.019 & 0.081 & 0.157 & 0.216 \\
\hline 300 & 0.500 & 0.532 & $\underline{0.567}$ & 0.539 & 0.042 & 0.204 & 0.481 & 0.463 \\
\hline 500 & 0.689 & 0.710 & 0.728 & $\underline{0.771}$ & 0.092 & 0.395 & 0.738 & 0.738 \\
\hline \multicolumn{9}{|c|}{$\hat{n}=n+1$} \\
\hline 30 & 0.0 & 0.006 & 0.039 & 0.002 & 0.0 & 0.0 & & 0.009 \\
\hline 50 & 0.001 & 0.005 & 0.053 & 0.001 & 0.0 & 0.0 & & 0.006 \\
\hline 100 & 0.004 & 0.015 & 0.043 & 0.003 & 0.0 & 0.0 & 0.023 & 0.004 \\
\hline 300 & 0.013 & 0.023 & 0.032 & 0.001 & 0.0 & 0.0 & 0.055 & 0.010 \\
\hline 500 & 0.021 & 0.027 & 0.038 & 0.0 & 0.0 & 0.0 & 0.058 & 0.013 \\
\hline
\end{tabular}

* The table shows the percentage cases where each criteria chose $n$, and the contiguous choices $n-1$ and $n+1$. The number of replications is 2000. The methods analyzed are the following: $S V C_{\Omega_{1}}$ from Bauer (2001), $S V C_{\Omega_{2}}$ that has the same structure but computed with the proposed weighting matrix $\Omega_{2}=\left(\tilde{Z}_{f} \tilde{Z}_{f}^{\prime}\right)^{-\frac{1}{2}}, N I D C$ which is as $S V C_{\Omega_{2}}$ but replacing the penalty term $C(T)=\log T / T$ by $H(T, i)=e^{-2} T^{-.9} i^{1.6} . A I C, S B C$ and $H Q$ are the information criteria due to Akaike (1976), Schwartz (1978) and Hannan and Quinn (1979) respectively. $\chi^{2}$ is the test proposed by Tsay (1989) with conventional significance level $5 \%$ and $M b C$ is the $\hat{n}$ value most often for each realization in the criteria collection, except $S V C_{\Omega_{1}}$. For each sample size, the largest relative frequency of hits is underlined. 
Table 5: System order estimates, M5 model ${ }^{\star}$.

$\operatorname{M5}(n=2):\left(1-0.4 B+0.3 B^{2}\right) z_{t}=(1-0.7 B) a_{t}, \quad a_{t} \sim \operatorname{iidN}(0,1)$.

\begin{tabular}{ccccccccc}
\hline $\mathrm{T}$ & $S V C_{\Omega_{1}}$ & $S V C_{\Omega_{2}}$ & $N I D C$ & $A I C$ & $S B C$ & $H Q$ & $\chi^{2}$ & $M b C$ \\
\hline \hline & \multicolumn{7}{c}{$\hat{n}=n-1$} \\
30 & 0.337 & 0.461 & 0.363 & 0.444 & 0.256 & 0.386 & 0.243 & 0.393 \\
50 & 0.589 & 0.588 & 0.360 & 0.483 & 0.481 & 0.510 & 0.478 & 0.524 \\
100 & 0.611 & 0.502 & 0.353 & 0.280 & 0.675 & 0.429 & 0.604 & 0.408 \\
300 & 0.168 & 0.129 & 0.115 & 0.015 & 0.204 & 0.051 & 0.152 & 0.060 \\
500 & 0.038 & 0.030 & 0.021 & 0.001 & 0.032 & 0.008 & 0.017 & 0.008
\end{tabular}

\begin{tabular}{ccccccccc}
\multicolumn{8}{c}{$\hat{n}=n$} \\
30 & 0.069 & 0.252 & $\underline{0.449}$ & 0.294 & 0.081 & 0.286 & 0.136 & 0.289 \\
50 & 0.186 & 0.321 & $\underline{0.516}$ & 0.459 & 0.146 & 0.402 & 0.229 & 0.394 \\
100 & 0.365 & 0.469 & 0.581 & $\underline{0.712}$ & 0.310 & 0.571 & 0.341 & 0.587 \\
300 & 0.802 & 0.837 & 0.835 & $\underline{0.973}$ & 0.796 & 0.947 & 0.802 & 0.929 \\
500 & 0.931 & 0.928 & 0.927 & $\underline{0.997}$ & 0.968 & 0.992 & 0.935 & 0.984
\end{tabular}

\begin{tabular}{ccccccccc}
\multicolumn{1}{c}{$\hat{n}=n+1$} \\
30 & 0.0 & 0.007 & 0.066 & 0.004 & 0.0 & 0.013 & 0.026 & 0.011 \\
50 & 0.0 & 0.008 & 0.092 & 0.003 & 0.0 & 0.014 & 0.023 & 0.009 \\
100 & 0.008 & 0.027 & 0.069 & 0.008 & 0.0 & 0.012 & 0.026 & 0.008 \\
300 & 0.028 & 0.031 & 0.044 & 0.011 & 0.0 & 0.015 & 0.021 & 0.011 \\
500 & 0.029 & 0.040 & 0.047 & 0.002 & 0.0 & 0.004 & 0.022 & 0.008 \\
\hline
\end{tabular}

* The table shows the percentage cases where each criteria chose $n$, and the contiguous choices $n-1$ and $n+1$. The number of replications is 2000 . The methods analyzed are the following: $S V C_{\Omega_{1}}$ from Bauer (2001), $S V C_{\Omega_{2}}$ that has the same structure but computed with the proposed weighting matrix $\Omega_{2}=\left(\tilde{Z}_{f} \tilde{Z}_{f}^{\prime}\right)^{-\frac{1}{2}}, N I D C$ which is as $S V C_{\Omega_{2}}$ but replacing the penalty term $C(T)=\log T / T$ by $H(T, i)=e^{-2} T^{-.9} i^{1.6} . A I C, S B C$ and $H Q$ are the information criteria due to Akaike (1976), Schwartz (1978) and Hannan and Quinn (1979) respectively. $\chi^{2}$ is the test proposed by Tsay (1989) with conventional significance level $5 \%$ and $M b C$ is the $\hat{n}$ value most often for each realization in the criteria collection, except $S V C_{\Omega_{1}}$. For each sample size, the largest relative frequency of hits is underlined. 
Table 6: System order estimates, M6 model ${ }^{\star}$.

$$
\begin{aligned}
& \operatorname{M6}(n=2):(\boldsymbol{I}+\boldsymbol{\Phi} B) \boldsymbol{Z}_{\boldsymbol{t}}=(\boldsymbol{I}+\boldsymbol{\Theta} B) \boldsymbol{a}_{\boldsymbol{t}}, \quad \boldsymbol{a}_{\boldsymbol{t}} \sim \operatorname{iidN}\left(\mathbf{0}, \boldsymbol{\Sigma}_{\boldsymbol{a}}\right) \\
& \boldsymbol{\Phi}=\left(\begin{array}{cc}
-0.4 & 0 \\
0 & -0.8
\end{array}\right) ; \boldsymbol{\Theta}=\left(\begin{array}{cc}
-0.7 & 0.5 \\
-0.3 & -0.7
\end{array}\right) ; \boldsymbol{\Sigma}_{\boldsymbol{a}}=\left(\begin{array}{cc}
1 & 0.5 \\
0.5 & 1
\end{array}\right)
\end{aligned}
$$

\begin{tabular}{ccccccccc}
\hline $\mathrm{T}$ & $S V C_{\Omega_{1}}$ & $S V C_{\Omega_{2}}$ & $N I D C$ & $A I C$ & $S B C$ & $H Q$ & $\chi^{2}$ & $M b C$ \\
\hline \hline \multicolumn{7}{c}{$\hat{n}=n-1$} \\
30 & 0.002 & 0.355 & 0.287 & 0.519 & 0.536 & 0.581 & 0.540 & 0.614 \\
50 & 0.068 & 0.491 & 0.358 & 0.517 & 0.702 & 0.632 & 0.699 & 0.665 \\
100 & 0.432 & 0.323 & 0.196 & 0.302 & 0.701 & 0.501 & 0.554 & 0.410 \\
300 & 0.007 & 0.002 & 0.002 & 0.005 & 0.249 & 0.078 & 0.054 & 0.012 \\
500 & 0.0 & 0.0 & 0.0 & 0.0 & 0.035 & 0.0 & 0.005 & 0.001
\end{tabular}

\begin{tabular}{ccccccccc}
\multicolumn{10}{c}{$\hat{n}=n$} \\
30 & 0.0 & 0.311 & $\underline{0.329}$ & 0.270 & 0.080 & 0.203 & 0.097 & 0.201 \\
50 & 0.001 & 0.354 & $\underline{\underline{0.429}}$ & 0.379 & 0.137 & 0.275 & 0.171 & 0.275 \\
100 & 0.199 & 0.611 & $\underline{0.655}$ & 0.617 & 0.290 & 0.470 & 0.414 & 0.555 \\
300 & $\underline{0.975}$ & 0.957 & 0.943 & 0.885 & 0.751 & 0.909 & 0.825 & 0.970 \\
500 & 0.982 & 0.972 & 0.958 & 0.896 & 0.965 & $\underline{0.992}$ & 0.851 & 0.984 \\
& \multicolumn{1}{c}{$\hat{n}=n+1$} & & & & & \\
30 & 0.0 & 0.183 & 0.224 & 0.081 & 0.008 & 0.031 & 0.007 & 0.033 \\
50 & 0.0 & 0.072 & 0.143 & 0.084 & 0.003 & 0.026 & 0.011 & 0.021 \\
100 & 0.001 & 0.047 & 0.112 & 0.071 & 0.001 & 0.027 & 0.024 & 0.035 \\
300 & 0.018 & 0.040 & 0.053 & 0.106 & 0.0 & 0.014 & 0.119 & 0.018 \\
500 & 0.018 & 0.028 & 0.040 & 0.098 & 0.0 & 0.002 & 0.148 & 0.015 \\
\hline
\end{tabular}

* The table shows the percentage cases where each criteria chose $n$, and the contiguous choices $n-1$ and $n+1$. The number of replications is 2000. The methods analyzed are the following: $S V C_{\Omega_{1}}$ from Bauer (2001), $S V C_{\Omega_{2}}$ that has the same structure but computed with the proposed weighting matrix $\Omega_{2}=\left(\tilde{Z}_{f} \tilde{Z}_{f}^{\prime}\right)^{-\frac{1}{2}}, N I D C$ which is as $S V C_{\Omega_{2}}$ but replacing the penalty term $C(T)=\log T / T$ by $H(T, i)=e^{-2} T^{-.9} i^{1.6}$. AIC, SBC and $H Q$ are the information criteria due to Akaike (1976), Schwartz (1978) and Hannan and Quinn (1979) respectively. $\chi^{2}$ is the test proposed by Tsay (1989) with conventional significance level $5 \%$ and $M b C$ is the $\hat{n}$ value most often for each realization in the criteria collection, except $S V C_{\Omega_{1}}$. For each sample size, the largest relative frequency of hits is underlined. 
Table 7: System order estimates, M7 model

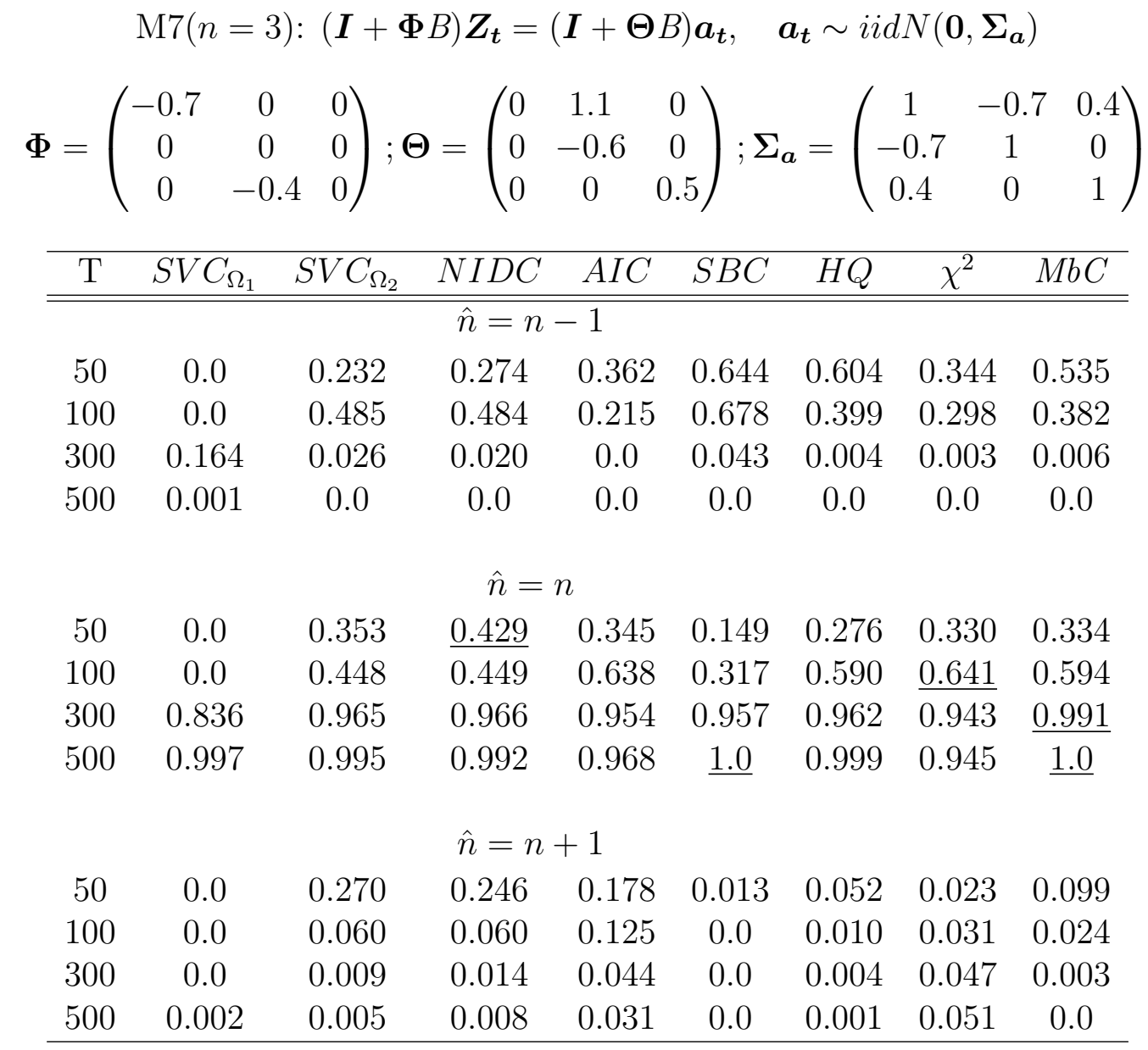

* The table shows the percentage cases where each criteria chose $n$, and the contiguous choices $n-1$ and $n+1$. The number of replications is 2000. The methods analyzed are the following: $S V C_{\Omega_{1}}$ from Bauer (2001), $S V C_{\Omega_{2}}$ that has the same structure but computed with the proposed weighting matrix $\Omega_{2}=\left(\tilde{Z}_{f} \tilde{Z}_{f}^{\prime}\right)^{-\frac{1}{2}}, N I D C$ which is as $S V C_{\Omega_{2}}$ but replacing the penalty term $C(T)=\log T / T$ by $H(T, i)=e^{-2} T^{-.9} i^{1.6}$. AIC, SBC and $H Q$ are the information criteria due to Akaike (1976), Schwartz (1978) and Hannan and Quinn (1979) respectively. $\chi^{2}$ is the test proposed by Tsay (1989) with conventional significance level $5 \%$ and $M b C$ is the $\hat{n}$ value most often for each realization in the criteria collection, except $S V C_{\Omega_{1}}$. For each sample size, the largest relative frequency of hits is underlined. 
Table 8: System order estimates, $M 8$ model ${ }^{\star}$.

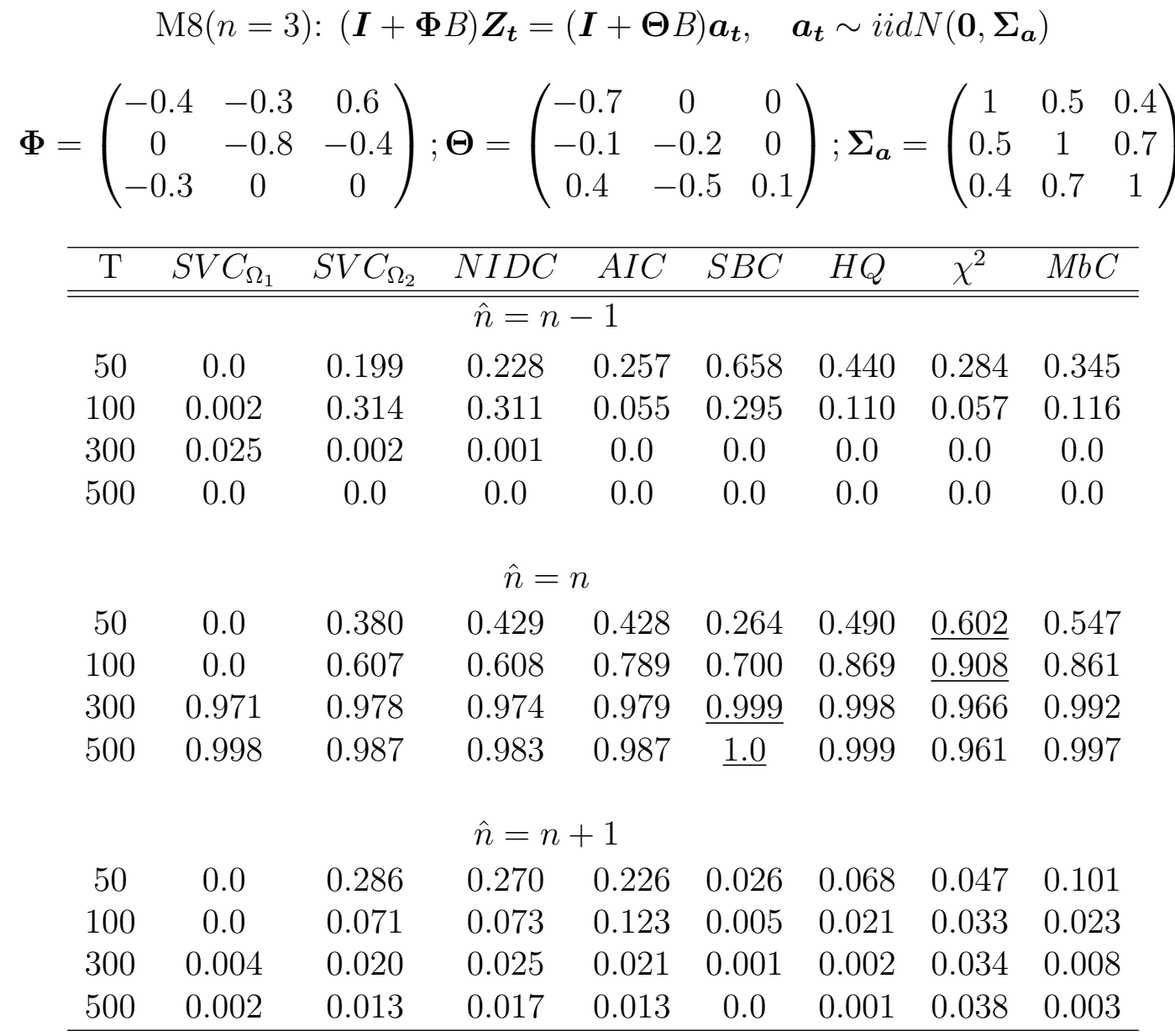

* The table shows the percentage cases where each criteria chose $n$, and the contiguous choices $n-1$ and $n+1$. The number of replications is 2000. The methods analyzed are the following: $S V C_{\Omega_{1}}$ from Bauer (2001), $S V C_{\Omega_{2}}$ that has the same structure but computed with the proposed weighting matrix $\Omega_{2}=\left(\tilde{Z}_{f} \tilde{Z}_{f}^{\prime}\right)^{-\frac{1}{2}}, N I D C$ which is as $S V C_{\Omega_{2}}$ but replacing the penalty term $C(T)=\log T / T$ by $H(T, i)=e^{-2} T^{-.9} i^{1.6}$. AIC, SBC and $H Q$ are the information criteria due to Akaike (1976), Schwartz (1978) and Hannan and Quinn (1979) respectively. $\chi^{2}$ is the test proposed by Tsay (1989) with conventional significance level $5 \%$ and $M b C$ is the $\hat{n}$ value most often for each realization in the criteria collection, except $S V C_{\Omega_{1}}$. For each sample size, the largest relative frequency of hits is underlined. 
Table 9: System orders estimates, M9 model.

\begin{tabular}{|c|c|c|c|c|c|c|c|c|}
\hline $\mathrm{T}$ & $S V C_{\Omega_{1}}$ & $S V C_{\Omega_{2}}$ & $N I D C$ & $A I C$ & $S B C$ & $H Q$ & $\chi^{2}$ & $M b C$ \\
\hline \multicolumn{9}{|c|}{$\hat{n}_{s}=n_{s}, s=4$} \\
\hline 30 & 0.453 & 0.634 & 0.734 & $\underline{0.816}$ & 0.569 & 0.747 & 0.470 & 0.690 \\
\hline 50 & 0.771 & 0.842 & 0.786 & $\underline{0.954}$ & 0.887 & 0.943 & 0.664 & 0.905 \\
\hline 100 & 0.925 & 0.897 & 0.819 & 0.952 & $\underline{0.996}$ & 0.985 & 0.888 & 0.961 \\
\hline 300 & 0.916 & 0.896 & 0.876 & 0.943 & $\underline{1.0}$ & 0.991 & 0.884 & 0.960 \\
\hline 500 & 0.920 & 0.907 & 0.883 & 0.943 & $\underline{1.0}$ & 0.996 & 0.890 & 0.962 \\
\hline \multicolumn{9}{|c|}{$\hat{n}_{r}=n_{r}, s=4$} \\
\hline 30 & 0.020 & 0.121 & $\underline{0.288}$ & 0.065 & 0.011 & 0.037 & 0.071 & 0.061 \\
\hline 50 & 0.041 & 0.117 & $\underline{0.314}$ & 0.068 & 0.009 & 0.031 & 0.104 & 0.071 \\
\hline 100 & 0.133 & 0.197 & $\underline{0.331}$ & 0.122 & 0.009 & 0.043 & 0.142 & 0.120 \\
\hline 300 & 0.415 & 0.457 & $\underline{0.497}$ & 0.443 & 0.021 & 0.152 & 0.467 & 0.400 \\
\hline 500 & 0.651 & 0.673 & 0.712 & 0.703 & 0.061 & 0.329 & $\underline{0.747}$ & 0.654 \\
\hline \multicolumn{9}{|c|}{$\hat{n}_{s}=n_{s}, s=12$} \\
\hline 100 & 0.969 & 0.972 & 0.960 & 0.984 & 0.992 & $\underline{0.996}$ & 0.460 & 0.988 \\
\hline 300 & 0.944 & 0.935 & 0.930 & 0.971 & $\underline{1.0}$ & 0.996 & 0.878 & 0.972 \\
\hline 500 & 0.946 & 0.938 & 0.931 & 0.942 & $\underline{1.0}$ & 0.998 & 0.894 & 0.975 \\
\hline \multicolumn{9}{|c|}{$\hat{n}_{r}=n_{r}, s=12$} \\
\hline 100 & 0.181 & 0.264 & $\underline{0.401}$ & 0.162 & 0.023 & 0.071 & 0.205 & 0.187 \\
\hline 300 & 0.495 & 0.529 & $\underline{0.562}$ & 0.487 & 0.040 & 0.192 & 0.536 & 0.476 \\
\hline 500 & 0.712 & 0.733 & $\overline{0.762}$ & 0.759 & 0.089 & 0.396 & $\underline{0.802}$ & 0.753 \\
\hline
\end{tabular}

* The table shows the percentage cases where each criteria chose $n_{s}$ and $n_{r}$. The number of replications is 2000. The methods analyzed are the following: $S V C_{\Omega_{1}}$ from Bauer (2001), $S V C_{\Omega_{2}}$ that has the same structure but computed with the proposed weighting matrix $\boldsymbol{\Omega}_{\mathbf{2}}=\left(\tilde{Z}_{f} \tilde{Z}_{f}^{\prime}\right)^{-\frac{1}{2}}, N I D C$ which is as $S V C_{\Omega_{2}}$ but replacing the penalty term $C(T)=\log T / T$ by $H(T, i)=e^{-2} T^{-.9} i^{1.6}$. AIC, SBC and $H Q$ are the information criteria due to Akaike (1976), Schwartz (1978) and Hannan and Quinn (1979) respectively. $\chi^{2}$ is the test proposed by Tsay (1989) with conventional significance level $5 \%$ and $M b C$ is the $\hat{n}$ value most often for each realization in the criteria collection, except $S V C_{\Omega_{1}}$. For each sample size, the largest relative frequency of hits is underlined. 
Table 10: System orders estimates, M10 model ${ }^{\star}$.

\begin{tabular}{|c|c|c|c|c|c|c|c|c|}
\hline $\mathrm{T}$ & $S V C_{\Omega_{1}}$ & $S V C_{\Omega_{2}}$ & $N I D C$ & $A I C$ & $S B C$ & $H Q$ & $\chi^{2}$ & $M b C$ \\
\hline \multicolumn{9}{|c|}{$\hat{n}_{36}=n_{36}$} \\
\hline 400 & 0.890 & 0.887 & 0.879 & 0.947 & 0.981 & $\underline{0.984}$ & 0.573 & 0.939 \\
\hline 600 & 0.902 & 0.888 & 0.866 & 0.955 & 0.997 & 0.992 & 0.764 & 0.952 \\
\hline 1000 & 0.846 & 0.832 & 0.867 & 0.949 & $\underline{0.998}$ & 0.991 & 0.760 & 0.930 \\
\hline \multicolumn{9}{|c|}{$\hat{n}_{9}=n_{9}$} \\
\hline 400 & 0.937 & 0.917 & 0.897 & 0.967 & $\underline{0.999}$ & 0.994 & 0.922 & 0.980 \\
\hline 600 & 0.931 & 0.920 & 0.896 & 0.972 & $\underline{0.999}$ & 0.995 & 0.989 & 0.996 \\
\hline 1000 & 0.929 & 0.918 & 0.942 & 0.980 & $\underline{1.0}$ & 0.997 & 0.989 & 0.996 \\
\hline \multicolumn{9}{|c|}{$\hat{n}_{3}=n_{3}$} \\
\hline 400 & 0.958 & 0.950 & 0.937 & 0.983 & $\underline{1.0}$ & 0.998 & 0.989 & 0.996 \\
\hline 600 & 0.950 & 0.946 & 0.934 & 0.987 & $\underline{1.0}$ & 0.998 & 0.988 & 0.996 \\
\hline 1000 & 0.932 & 0.928 & 0.944 & 0.985 & $\underline{1.0}$ & $\underline{1.0}$ & 0.988 & 0.992 \\
\hline \multicolumn{9}{|c|}{$\hat{n}_{1}=n_{1}$} \\
\hline 400 & 0.974 & 0.969 & 0.962 & 0.994 & $\underline{1.0}$ & 0.999 & 0.995 & 0.997 \\
\hline 600 & 0.981 & 0.979 & 0.971 & 0.996 & $\underline{1.0}$ & $\underline{1.0}$ & 0.993 & 0.999 \\
\hline 1000 & 0.963 & 0.959 & 0.970 & 0.986 & $\underline{1.0}$ & $\underline{1.0}$ & 0.989 & 0.997 \\
\hline
\end{tabular}

* The table shows the percentage cases where each criteria chose $n_{s}, s=3,6,9$ and $n_{r}$. The number of replications is 2000. The methods analyzed are the following: $S V C_{\Omega_{1}}$ from Bauer (2001), $S V C_{\Omega_{2}}$ that has the same structure but computed with the proposed weighting matrix $\boldsymbol{\Omega}_{\mathbf{2}}=\left(\tilde{Z}_{f} \tilde{Z}_{f}^{\prime}\right)^{-\frac{1}{2}}, N I D C$ which is as $S V C_{\Omega_{2}}$ but replacing the penalty term $C(T)=\log T / T$ by $H(T, i)=e^{-2} T^{-.9} i^{1.6}$. AIC, $S B C$ and $H Q$ are the information criteria due to Akaike (1976), Schwartz (1978) and Hannan and Quinn (1979) respectively. $\chi^{2}$ is the test proposed by Tsay (1989) with conventional significance level $5 \%$ and $M b C$ is the $\hat{n}$ value most often for each realization in the criteria collection, except $S V C_{\Omega_{1}}$. For each sample size, the largest relative frequency of hits is underlined. 